\title{
Narrativa
}

narrativa

Nuova serie

43 | 2021

La fantascienza nelle narrazioni italiane

ipercontemporanee

\section{La fantascienza nelle narrazioni italiane ipercontemporanee}

Daniele Comberiati e Luca Somigli

\section{(2) OpenEdition}

\section{Journals}

Edizione digitale

URL: https://journals.openedition.org/narrativa/416

DOI: $10.4000 /$ narrativa.416

ISSN: 2804-1224

\section{Editore}

Presses universitaires de Paris Nanterre

\section{Edizione cartacea}

Data di pubblicazione: 1 décembre 2021

Paginazione: 7-17

ISBN: 978-2-84016-485-2

ISSN: $1166-3243$

Notizia bibliografica digitale

Daniele Comberiati e Luca Somigli, «La fantascienza nelle narrazioni italiane ipercontemporanee»,

Narrativa [Online], 43 | 2021, online dal 01 novembre 2021, consultato il 03 novembre 2022. URL:

http://journals.openedition.org/narrativa/416 ; DOI: https://doi.org/10.4000/narrativa.416

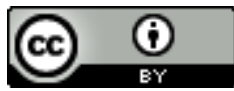

Creative Commons - Attribuzione 4.0 Internazionale - CC BY 4.0

https://creativecommons.org/licenses/by/4.0/ 


\title{
La fantascienza nelle narrazioni italiane \\ ipercontemporanee
}

\begin{abstract}
RiASSUNTO
Se la fantascienza italiana è per molto tempo stata vista come genere minore o "derivativo" rispetto ai modelli anglosassoni, le produzioni iper-contemporanee mostrano al contrario una grande originalità e la capacità di approfondire alcuni dei temi più importanti della società contemporanea. In questo testo introduttivo sono presentati inoltre gli articoli del volume, che spaziano fra diversi temi ma mostrano tutti una particolare capacità di riflettere sulla realtà circostante.
\end{abstract}

\section{RÉSUMÉ}

Si la science-fiction italienne a longtemps été considérée comme un genre mineur ou "dérivé" par rapport aux modèles anglo-saxons, les productions hyper-contemporaines font preuve au contraire d'une grande vivacité et d'une capacité à approfondir certains des thèmes les plus importants de la société contemporaine. Dans ce texte introductif, sont également présentés les articles du volume, qui abordent des thématiques différentes mais qui montrent tous la capacité de réfléchir sur la réalité environnante.

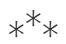

Nel contesto letterario italiano, a causa di varie ragioni che vanno dalla divisione crociana fra cultura "alta" e cultura "bassa" all'erronea valutazione del genere come esclusivamente "derivativo" delle produzioni anglosassoni, la fantascienza ha avuto difficoltà a legittimarsi ed essere legittimata nel corso della seconda metà del Novecento. Considerata per lo più narrativa popolare - con tutta l'ambiguità e le contraddizioni che la definizione rivela -, anche quando è stata impiegata da autori affermati (si veda il caso, esemplificativo, di Calvino, 
ma in parte anche quello di Buzzati) ${ }^{1}$, non è riuscita a essere considerata "vera" letteratura, aprendo però, forse anche suo malgrado, una serie di riflessioni più ampie e profonde sul ruolo della letteratura nella società, sul ricorso a generi cosiddetti bassi per descrivere e rappresentare alcuni aspetti della realtà, sulla formazione e circolazione di un immaginario che si pone al tempo stesso nazionale e transnazionale, sulla ricezione e la rielaborazione delle convenzioni del genere che mostrano, in ambito italiano, una vitalità e una creatività troppe volte trascurate.

Anche a causa dei cambiamenti che hanno investito le nostre società, dal punto di vista ecologico, e delle trasformazioni delle rappresentazioni della realtà - dall'arrivo e diffusione di internet e dei social media, fino al successo di narrazioni seriali distopiche, senza trascurare ovviamente l'emergenza climatica e la rapidità dei nuovi scambi globali -, negli ultimi anni le produzioni letterarie di fantascienza in Italia si sono moltiplicate, per numero e popolarità. Autori e autrici hanno contribuito ad ampliare considerevolmente il corpus di un genere che per molti anni in Italia è stato considerato minore. Citeremo, sapendo di dimenticarne molte, opere come Anna di Niccolò Ammaniti (2015), La festa nera di Violetta Bellocchio (2018), Nel grande vuoto di Adil Bellafqih (menzione speciale al Premio Calvino 2019), Avrai i miei occhi di Nicoletta Vallorani (2019), L'isola delle madri di Maria Rosa Cutrufelli (2020), Configuražione Tundra di Elena Giorgiana Mirabelli (2020).

Si tratta di testi molto diversi fra loro, per i quali è necessario utilizzare una definizione aperta del genere fantascientifico, sulla linea di quanto già teorizzato in Italia da un autore fondamentale per l'evoluzione della fantascienza negli anni Sessanta e Settanta come Lino Aldani, che è stato al tempo stesso scrittore, critico e animatore culturale del genere ${ }^{2}$. Tale riflessione è stata poi ripresa tra gli altri da John Rieder nel 2010, che si è interessato alla molteplicità di definizioni che si possono dare alla fantascienza, mettendo in discussione categorizzazioni riduttive che hanno solo la funzione di limitarne la portata innovativa e creativa $^{3}$. In generale, a partire da queste due posizioni critiche, possiamo osservare come non sia tanto importante definire la fantascienza e circoscriverla all'interno di confini, quanto comprendere in che modo l'impiego di un genere

1. Cfr. Calvino Italo, Le cosmicomiche, Torino, Einaudi, 1965; Buzzati Dino, Il grande ritratto, Milano, Bompiani, 1960.

2. Aldani Lino, La fantascienza, Piacenza, La Tribuna, 1962.

3. Rieder John, "On Defining Science-Fiction or Not. Genre, Theory, Science-Fiction, and History", in Science Fiction Studies, vol. 37, n. 2, pp. 191-209. 
poroso e aperto alla rappresentazione delle innovazioni tecnologiche possa rivelarsi adatto per descrivere una realtà in costante mutazione.

Anche in ambito critico e accademico sono stati ultimamente pubblicati nuovi importanti contributi, fra cui, per non citarne che alcuni, il volume monografico di Science Fiction Studies a cura di Arielle Saiber, Salvatore Proietti e Umberto Rossi (2015), lo studio sulla nascita e il successo della fantascienza in Italia di Pier Paolo Antonello (2008), i testi a quattro mani di Simone Brioni e Daniele Comberiati (2019 e 2020), la creazione della collana "Fantascienza e Società", a cura di Domenico Gallo, per l'editore Mimesis nel 2012, i lavori di Giulia Iannuzzi ${ }^{4}$. Parallelamente, oltre al lavoro costante della storica collana "Urania" di Mondadori, è nata nel 2018 la collana "Altrove", dedicata espressamente alle distopie italiane, inaugurata da Chiarelettere, mentre gli editori Delos e Zona 42 propongono un catalogo interamente dedicato al genere che include ormai numerosi testi italiani.

Proprio a partire da queste nuove e fervide esperienze, è possibile notare come oggi la fantascienza dialoghi con alcuni dei campi teorici più interessanti del contesto contemporaneo, quali il postumanesimo e il transumanesimo, l'ecocritica, la collassologia, oltre ovviamente a mantenere un confronto aperto con gli studi di genere, culturali e postcoloniali. Anche la presenza di sottogeneri quali il cyberpunk, il solarpunk o lo steampunk, che cercano di immaginare un futuro al di là di un approccio troppo facilmente pessimista contribuiscono alla ricchezza degli studi critici e delle produzioni fantascientifiche attuali, così come le narrazioni post-apocalittiche, catastrofistiche, utopiche e distopiche che in ambito italiano riprendono una tradizione di fantascienza sociologica che risale agli anni Sessanta ed è ormai consolidata, ma che nelle produzioni contemporanee è stata attualizzata per descrivere i mutamenti delle società in cui autori e autrici operano.

Ciononostante, il campo di studi rimane per molti versi ancora inesplorato, oppure analizzato a partire da un canone interno che fatica a rinnovarsi:

4. Saiber Arielle, Proietti Salvatore, Rossi Umberto (a cura di), Science Fiction Studies, vol. 42, n. 2, 2015; Brioni Simone, Comberiati Daniele, Italian Science Fiction. The Other in Literature and Film, New York, Palgrave Macmillan, 2019; BRIONI Simone, Comberiati Daniele, Ideologia e rappresentazione. Percorsi attraverso la fantascienza italiana, Milano, Mimesis, 2020; Antonello Pierpaolo, "La nascita della fantascienza in Italia. Il caso Urania", in Schnapp Jeffrey, Scarpeldini Elena (a cura di), ItaliAmerica. Le origini dell'americanismo in Italia, Milano, Il Saggiatore, 2008, pp. 99-123; IANNuZzI Giulia, Fantascienza italiana. Riviste, autori, dibattiti, dagli anni Cinquanta agli anni Settanta, Milano, Mimesis, 2014; EAD., Distopie, viaggi, allucinazioni. Fantascienza italiana contemporanea, Milano, Mimesis, 2016. 
l'iper-contemporaneità, infatti, sembra aver trovato proprio nella rappresentazione fantascientifica una chiave particolarmente adatta a raccontarsi e a mettere in luce i propri risvolti ideologici e politici. Molti temi restano ancora da approfondire: la questione del "genere" (inteso come gender) nella letteratura italiana fantascientifica contemporanea ${ }^{5}$; la transmedialitá e dunque la relazione delle narrazioni fantascientifiche con le arti visuali come il cinema, le serie televisive o il fumetto; il rapporto con il canone straniero, soprattutto (ma non solo) anglofono e francofono; i temi della disgregazione dello stato nazionale e delle migrazioni; i danni causati dall'inquinamento e in ultimo dalla pandemia del 2020. Sono solo alcune indicazioni di tematiche che la fantascienza ha saputo raccontare con tempestività e originalità; queste e altre meritano riflessioni critiche approfondite. Il numero in questione si propone proprio di indagare queste nuove narrazioni, che rendono oggi la fantascienza uno dei generi più interessanti nella produzione italiana contemporanea e uno degli ambiti teorici più vivaci.

In "La fantascienza italiana dalla prospettiva degli studi sulla traduzione e postcoloniali", il saggio che apre il volume, Simone Brioni torna sulla questione della presunta natura derivativa della fantascienza italiana da prototipi d'importazione soprattutto anglosassone. Prendendo come punto di riferimento l'impostazione teorica secondo cui la traduzione non è la semplice riproduzione di secondo grado di un originale ma un'operazione di riscrittura che situa il nuovo testo in un diverso contesto storico e culturale, Brioni sottolinea come il confronto con i modelli anglosassoni e la loro riscrittura possa essere interpretata nei termini di quella che Homi Bhabha chiama "ibridazione", cioè un processo complesso di ripresa e di resistenza alle strutture ideologiche e commerciali degli originali che condivide molto con la dinamica che regge il rapporto tra culture subalterne e culture dominanti in un contesto coloniale o postcoloniale. Il relativo straniamento imposto dal modello straniero (a cui va aggiunto, nel nostro caso, lo "straniamento cognitivo" costitutivo del genere fantascientifico, secondo la nota lezione di Darko Suvin) ${ }^{6}$ da un lato rende possibile articolare prospettive inedite per la cultura che se ne appropria, dall'altro permette di mettere in luce e rendere visibili (e quindi passibili di critica) i presupposti e gli assunti culturali del modello stesso. 2021.

5. Cfr. a tale proposito CurTi Lidia (a cura di), Femminismi futuri, Roma, Iacobelli,

6. Suvin Darko, Metamorphoses of Science Fiction: On the Poetics and History of a Literary Genre, New Haven, Yale University Press, 1979. 
Non è difficile identificare modelli americani per il sottogenere della "storia alternativa" o "ucronia", per usare il termine più corrente: pochi romanzi di fantascienza hanno avuto la fortuna critica di The Man in the High Castle (1962) di Philip K. Dick, che immagina un mondo in cui le potenze dell'Asse hanno vinto la Seconda Guerra Mondiale, mentre con The Plot against America (2004), Philip Roth, un autore altrimenti estraneo alla letteratura di genere, ha dimostrato il potenziale dell'ucronia come strumento per mettere in luce i lati oscuri della storia recente. Un'operazione simile è al cuore della trilogia di Enrico Brizzi iniziata nel 2008 con L'inattesa piega degli eventi, e di cui si occupa il saggio di Umberto Rossi ("Contro-passato prossimo: genesi di una storia alternativa in La nostra guerra di Enrico Brizzi'). La scrittura di genere in Italia non è aliena dal servirsi di contro-storie come strategia interpretativa di fronte all'opacità della storia ufficiale - basti pensare all'ampia produzione giallistica e noir sui cosiddetti "misteri d'Italia" già praticata da un autore come Loriano Macchiavelli fin dagli anni '70 - e l'ucronia si rivela particolarmente adatta allo scopo. In Brizzi, lo straniamento ucronico produce un mondo diverso eppure curiosamente familiare, in cui il fascismo post-bellico sembra condividere elementi ideologici, strategie politiche e addirittura personale dirigente con la Democrazia Cristiana del nostro passato, a sottolineare la continuità, nel mondo "vero", tra ventennio e Italia post-bellica. Vale anche la pena di rimarcare, a riprova di come appunto il recupero di modelli stranieri non sia un semplice gesto di subalternità culturale, come la fantascienza ucronica italiana sia diventata, agli inizi degli anni 2000, uno dei terreni su cui si è combattuta la battaglia per "sdoganare" e normalizzare movimenti politici e culturali scopertamente neo-fascisti: la trilogia di Brizzi è anche una risposta all'ucronia del fascismo trionfante proposta con non poca enfasi retorica dalla trilogia di Occidente di Mario Farnetti. L'ucronia è, insieme all'autofiction, il genere a cui afferisce, almeno per quanto riguarda la fabula, anche Medium di Giuseppe Genna (2007), romanzo altrimenti difficilmente catalogabile e non a caso collocato da Wu Ming 1 tra gli "oggetti narrativi non identificati" in New Italian Epic (2008). In "Hantologia e fantarcheologia in Medium di Giuseppe Genna", Fabio Camilletti propone una lettura del romanzo in chiave hantologica, di ritorno fantasmatico di un passato trasfigurato in chiave fantastica. Di fronte alla celebrazione prematura della "fine della Storia" con la caduta del Muro di Berlino, secondo l'arcinota formula di Francis Fukuyama, Medium mette in scena il ritorno spettrale del Padre sia come figura biografica che come orizzonte ideologico-culturale in una forma straniata che restituisce a entrambi la possibilità di una pienezza di senso. 
I casi di Brizzi e Genna forniscono un'ulteriore prova del fatto che la fantascienza si è ormai definitivamente emancipata dallo statuto di letteratura popolare $\mathrm{o}$, peggio, paraletteratura, ed è né più né meno che una modalità romanzesca a cui attingere per assolvere ai più diversi progetti letterari, dalla critica sociale allo scavo nelle proprie nevrosi. In questo spazio in cui la distinzione tra letteratura di genere e non di genere è non solo abolita ma è priva di senso si collocano diversi dei contributi nel presente volume. In "L'originale di Paolo. La fantascienza nell'opera narrativa di Paolo Zanotti”, Stefano Lazzarin ripercorre l'opera narrativa dello scrittore scomparso prematuramente nel 2012, e mostra come l'uso di situazioni e tropi fantascientifici e, in Il Testamento Disney (postumo, 2013), addirittura l'uso metadiscorsivo della SF come lente attraverso cui leggere il mondo, servano a dare forma narrativa al vero tema della scrittura di Zanotti: "il felice e tragico mondo dell'infanzia e l'impossibile transizione dall'infanzia all'età adulta". L'infanzia e le sue angosce sono al centro anche della narrativa di Michele Mari di cui si occupa Stefania Lucamante nel saggio “'Un'orrenda vita da vivere': La leggenda privata di Michele Mari fra autofiction e SF". Fantascienza, horror, gotico, fumetto - la biblioteca di una vita per un autore onnivoro come Mari - forniscono gli elementi per assemblare una narrazione autofittiva in cui lo scarto rispetto al reale che è costitutivo della letteratura di genere serve precisamente a dare forma a ciò che non si può guardare direttamente, come appunto i traumi dell'infanzia.

Con il già citato L'isola delle madri, di cui scrivono Ramona Onnis e Manuela Spinelli ("Dalla Madre alle madri? Fra distopia, mito e realtà"), Maria Rosa Cutrufelli sceglie il terreno della SF e più specificamente del sotto-genere dell'eco-distopia per un romanzo in cui la rappresentazione di un futuro devastato dalla crisi ecologica - il novum fantascientifico propriamente detto - serve da sfondo su cui proiettare una serie di tematiche riprese dalla tradizione della narrativa femminista, dal controllo del corpo della donna e della riproduzione alla maternità e alle forme di solidarietà femminile.

Un sottogenere particolarmente impiegato nelle narrazioni italiane contemporanee - anche in tal caso, riprendendo il saggio iniziale di Brioni, in parte attraverso riscritture di modelli stranieri, soprattutto anglosassoni, in parte proseguendo una precisa linea narrativa nazionale, da far risalire almeno agli anni Sessanta e alle riflessioni ambientaliste di Dino Buzzati, Emilio De Rossignoli e Lino Aldani ${ }^{7}$ - risulta appunto senz'altro l'eco-distopia, simbolo di una

7. Buzzati Dino, Il grande ritratto, cit.; De Rossignoli Emilio, H come Milano, Milano, Garzanti, 1965; Aldani Lino, Quando le radici, Piacenza, La Tribuna, 1977. 
narrazione catastrofista o post-catastrofista, che si declina alle volte come estremamente pessimista e altre come foriera di una possibilità (l'ultima?) di ricostruzione del genere umano. È il caso ad esempio del lavoro di Pierpaolo Antonello "Post-umano, troppo post-umano: Sirene di Laura Pugno". L'articolo, che prendendo in esame il romanzo di Pugno del 2006 si rivela prezioso anche per il consolidamento del canone fantascientifico contemporaneo cui accennavamo in precedenza, ha il merito di introdurre due importanti prospettive di studio delle narrazioni italiane di SF. Innanzitutto l'autore affronta la teoria del post-umano la quale, pur decisiva nella fantascienza globale contemporanea, viene talvolta impiegata nel contesto italiano (e non solo) con un ottimismo eccessivo rispetto ad un ipotetico futuro inter-specie e in cui l'essere umano sarà capace di ibridarsi e vivere con gli altri elementi del pianeta. In secondo luogo, l'articolo di Antonello affronta la questione di genere, anch'essa ormai consolidata all'interno della produzione nazionale (basti pensare ai casi, per non citarne che alcuni, di Roberta Rambelli, Gilda Musa e Luce D’Eramo) e visibile sia dal cospicuo numero di scrittrici contemporanee, sia dalle tematiche affrontate, attraverso le quali il futuro dell'umanità è spesso associato ad un ripensamento se non addirittura a un capovolgimento delle relazioni fra i generi o ad una riflessione sulla modifica dell'attuale categoria di "genere". Sirene è anche al centro delle riflessioni di Amélie Aubert-Noël ("Ibridità testuale e ibridità creaturale in Sirene di Laura Pugno"), in cui la stessa struttura ibrida del testo, che afferisce a più generi letterari (manga, racconto post-apocalittico, mito e fantastico) si ripercuote sulla rappresentazione degli esseri mitici immaginati dalla scrittrice, segno di una dualità, anzi di una molteplicità, che sono anche il simbolo della porosità del genere, o piuttosto dei generi utilizzati.

La riflessione sul genere e l'assunzione di un impiego in senso "allargato" della fantascienza, in linea con alcune delle principali teorizzazioni italiane e straniere (pensiamo in particolare ancora ad Aldani per il caso italiano ${ }^{8}$ ), è alla base anche del saggio di Hanna Serkowska e Aleksandra Pławska, nel quale la lettura di Sirene e di altre opere di Pugno è effettuata attraverso la specula di due sottogeneri: da una parte la "speculative-evolution fiction", sorta di prosecuzione del romanzo utopico distopico con un'attenzione alle possibili evoluzioni del genere umano, dall'altra la "quantum-actualistic fiction", che utilizza la funzione quantica per strutturare percorsi narrativi non lineari e che non seguono dunque le leggi della meccanica e della statistica.

8. Aldani Lino, La fantascienza, cit. 
Un primo corpus sulla letteratura eco-distopica italiana è in seguito tracciato da Marco Malvestio nell'articolo "Sognando la catastrofe. L'eco-distopia italiana del ventunesimo secolo". L'autore, che prende in considerazione solo i testi in cui appaiono evidenti componenti di critica ecologica, tracciando inoltre nell'introduzione una breve panoramica del sottogenere e della categoria critica a esso associata, individua almeno quattordici opere pubblicate a partire dal 2007, per un elenco che ovviamente non può essere che parziale e ancora in fieri. Fra queste naturalmente ancora Sirene di Laura Pugno, ma anche Anna di Niccolò Ammaniti (altro testo che ritorna spesso nella costruzione di un canone del sottogenere), Cinacittà di Tommaso Pincio e Qualcosa, là fuori di Bruno Arpaia, uno dei romanzi di riferimento dell'eco-distopia. All'ambito eco-distopico è infine riconducibile anche il sottogenere della "retrotopia", della cui declinazione italiana si occupa Florian Mussgnug nel saggio "Speculazioni ecologiche: impegno e retrotopia nel romanzo italiano contemporaneo". Dopo una panoramica molto articolata del dibattito teorico su eco-critica e narrativa speculativa, Mussgnug rileva come, a differenza di altre narrazioni sull'apocalissi climatica, la retrotopia si sia caratterizzata da uno sguardo retrospettivo nostalgico verso un passato pre-industriale idealizzato, e spesso sembri proporre come punto d'approdo l'utopia di un ritorno alla natura che rischia di apparire soverchiamente semplificatoria e regressiva.

Se uno degli aspetti più interessanti della SF in generale e di quella italiana in particolare è rappresentato dall'aderenza e talvolta dall'anticipazione delle evoluzioni della realtà circostante, l'eco-distopia diventa certamente una tipologia di narrazione privilegiata, così come diviene fondamentale indagare quali siano le strategie narrative attraverso le quali i testi di maggior impatto su pubblico e critica sono costruiti. A tale proposito il contributo a quattro mani di Monica Jansen e Claudio Milanesi ("Eva e Avrai i miei occhi: tracciare e ricomporre le mappe sulla pelle. Conversation pieces") ci introduce alla scrittura di Nicoletta Vallorani, una delle autrici più interessanti del panorama italiano contemporaneo. L'articolo ci permette di comprendere inoltre alcune delle caratteristiche principali della produzione odierna: l'ibridazione fra i generi, tra l'altro già menzionata per quanto riguarda l'opera di Laura Pugno; l'individuazione della postmodernità come momento chiave in cui la divisione fra cultura "alta" e "bassa" è definitivamente crollata, lasciando ad autori e autrici la possibilità di utilizzare riferimenti provenienti da contesti molto diversi fra loro; la funzione fondamentale della transmedialità, da intendere come una possibile prosecuzione dell'opera in altri campi di espressione - come è il caso per il fumetto, per il cinema o anche per le serie televisive, di cui Anna (2020), il cui coregista è lo 
stesso Ammaniti, rappresenta forse il caso più eclatante -, ma anche come percorso di origine e ispirazione, basti pensare ai film che hanno influenzato la scrittura di Vallorani. Vale qui la pena di sottolineare come le tematiche eco-distopiche e post-apocalittiche al centro di molta della narrativa fin qui esaminata si intreccino strettamente con la questione del "gender": non a caso, sia Sirene sia i romanzi di Vallorani mettono la devastazione ambientale in relazione all'androcentrismo della cultura occidentale e hanno al loro centro figure che problematizzano i binarismi di genere (nonché di specie e di umano/artificiale). Di "Fantascienza femminista" si occupa il saggio di Giuseppe Carrara in cui, partendo proprio da Vallorani e dall'identificazione di una serie di ambiti tematici quali il corpo, l'identità e il linguaggio di cui da sempre si è occupata la narrativa femminista, l'autore inizia a delineare un canone che comprende anche Violetta Bellocchio e Veronica Raimo, autrici di opere inquadrabili anch'esse nel campo del romanzo utopico o distopico.

Il filone eco-distopico viene in parte analizzato anche da Simona Micali ("I bambini dell'apocalisse. Racconti della fine e di nuovi inizi nella fantascienza italiana degli anni Duemila") che, a partire dall'approccio postumano, evidenzia da una parte come sia stato proprio il racconto post-apocalittico (del quale traccia anche una breve storia) ad avere sdoganato e in parte legittimato la fantascienza in ambito italiano, e dall'altra come molte di queste narrazioni mettano in scena bambini e adolescenti, quasi a voler presagire un radicale cambiamento di paradigma del mondo che conosciamo a partire dalle prossime generazioni. Tale pars construens delle narrazioni post-apocalittiche italiane - in vero meno analizzata rispetto alle visioni catastrofiste - , si inserisce in una riscrittura del modello classico apocalittico/palingenetico, ma al tempo stesso si sforza di proporre al lettore e alla lettrice ipotesi concrete di nuove umanità.

A tale proposito il contributo di Valentina Fulginiti - "Dei bambini non si sa niente? L'infanzia come altrove distopico in Anna (Ammaniti), Bambini Bonsai (Zanotti), La terra dei figli (Gipi) e L'uomo verticale (Longo)" - si interroga giustamente sul ruolo privilegiato di bambini e adolescenti nelle narrazioni distopiche, cercando di mettere in luce come le rappresentazioni di società senza adulti forniscano agli autori lo spunto per una riflessione sulle relazioni fra natura umana e patto sociale, indagando i due estremi cui accennavamo al principio del nostro discorso: una distopia catastrofista e senza speranza o, dall'altro lato, una visione eco-critica che possa fungere da ancora di salvezza per il genere umano e che gli consenta di ripensarsi e ricostruirsi.

In effetti il tema dei bambini - come personaggi dei romanzi di fantascienza citati, ma anche come pubblico al quale queste opere si rivolgono - è un ulteriore 
filone rilevante del corpus di SF italiana. Beatrice Laghezza a tale proposito si interroga proprio sulla fantascienza italiana Young Adults, ragionando in termini di crossover e di transmedialità. Una fantascienza che rimanda, talvolta come visto in modo esplicito, ad altri linguaggi (cinema, televisione, videogames, fumetto), ma che al tempo stesso spesso dispiega in modo metanarrativo l'oggetto libro, considerato in diverse narrazioni che l'autrice prende in esame come l'elemento che salverà l'essere umano dall'estinzione.

Una vocazione transmediale caratterizza anche il progetto del movimento connettivista, di cui dà conto Arielle Saiber in un'ampia intervista a uno dei suoi fondatori, Giovanni De Matteo, preceduta da una nota critica e di poetica. Se il termine "connettivismo" è di derivazione letteraria e rimanda a un autore della fantascienza classica, A. E. van Vogt, la sua ripresa in questo contesto vuole evocare invece quell'incrocio di forme di sapere e di produzione culturale il cui potenziale è stato moltiplicato dalle nuove piattaforme mediatiche, internet prima su tutte. L'intersezionalità è dunque insita nel nome del movimento stesso, teso alla ricerca, come scrive Saiber, di "un tentativo di sintesi e ibridazione: tra linguaggi e forme espressive diverse, tra mondi in opposizione e saperi sbrigativamente considerati inconciliabili, tra generi reputati statici e cristallizzati”. Nel ripercorrere la storia del movimento dal lancio del manifesto fondativo nel 2004, Saiber ne sottolinea la capacità aggregativa intorno a case editrici e progetti online, in un continuo processo di sperimentazione che, pur tenendo la letteratura al suo centro, spazia dalle arti figurative alla musica.

Una riflessione sulla transmedialità e sulle narrazioni inter-generazionali è presente infine nel saggio di Anna Chiara Palladino consacrato a uno dei fenomeni cinematografici italiani degli ultimi anni, Lo chiamavano Jeeg Robot di Gabriele Mainetti (2016). In tal caso il regista ibrida almeno tre tradizioni culturali (la SF italiana, gli anime giapponesi, i film di supereroi americani) creando un prodotto al tempo stesso sequenziale e innovativo, capace di parlare a un pubblico eterogeneo per età e interessi culturali.

E forse non è un caso che il recente interesse che si riscontra verso la fantascienza italiana - per anni relegata soprattutto ai film di "serie B" o alle collane specializzate - sia coevo al cambiamento di gusto di un pubblico che attraverso altri linguaggi (in particolare le serie televisive) conosce ormai le convenzioni del genere. $\mathrm{Al}$ tempo stesso, ciò che colpisce nel corpus di cui questo numero cerca di fornire un esempio, è la grande varietà di strategie narrative e sottogeneri: l'interesse per la fantascienza italiana, oltre che per la sua capacità di analizzare i conflitti della contemporaneità (dalla crisi della democrazia al riscaldamento climatico, dai rischi della digitalizzazione alle disuguaglianze di 
genere, razza e classe), è fornito anche dalla ricchezza dei punti di vista. Forse in tal caso il fatto che la fantascienza sia stata considerata per diverso tempo un genere minore ha permesso agli autori e alle autrici che ne hanno utilizzato le convenzioni una particolare libertà, scegliendo modelli narrativi stranieri, decostruendo e ricostruendo costantemente il canone, ibridandola con altre tipologie narrative. La produzione contemporanea, in letteratura ma non solo, conferma questa tendenza, oltre a mostrare una relazione sempre più stretta con altri linguaggi.

Daniele Comberiati

Université Paul-Valéry Montpellier 3

Luca SomigLI

University of Toronto 
